\title{
Goals of Clinical Ethics Support: Perceptions of Dutch Healthcare Institutions
}

\author{
L. Dauwerse - T. A. Abma • B. Molewijk • \\ G. Widdershoven
}

Published online: 13 September 2011

(C) The Author(s) 2011. This article is published with open access at Springerlink.com

\begin{abstract}
In previous literature, ethicists mention several goals of Clinical Ethics Support (CES). It is unknown what key persons in healthcare institutions see as main__and sub-goals of CES. This article presents the goals of CES as perceived by board members and members of ethics support staff. This is part of a Dutch national research using a mixed methods design with questionnaires, focus groups and interviews. Quantitative and qualitative data were analyzed and combined in an iterative process. Four main clusters of goals were found: 1) encouraging an ethical climate, 2) fostering an accountable and transparent organization, 3) developing professionalism and a final goal, overarching the previous three, 4) good care. Most important sub-goals of CES were: attention for ethical issues, raising awareness of ethical issues, fostering ethical reflection and supporting employees. The article ends with a discussion on the desirability to further operationalize the general goal of good care, the context-boundedness of our findings and the need to relate goals of CES to the features of organizational cultures to further improve the integration of CES in healthcare institutions.
\end{abstract}

\section{Introduction}

Previous literature shows that healthcare institutions increasingly acknowledge the value of Clinical Ethics Support (CES), and structurally integrate clinical ethics in their organization through CES ranging from ethics consultation and ethics

\footnotetext{
L. Dauwerse $(\varangle)$ - T. A. Abma · B. Molewijk · G. Widdershoven Department of Medical Humanities, The EMGO Institute for Health and Care Research, VU University Medical Centre, Van der Boechorststraat 7, 1081 BT Amsterdam, The Netherlands e-mail: 1.dauwerse@vumc.nl

B. Molewijk

GGNet, Institute for Mental Health Care, Warnsveld, The Netherlands
} 
committees to moral case deliberation $[7,8,16,17]$. At the same time, the goals of CES are often not, or not clearly, formulated in practice. Slowther [16] indicates that the aims of CES are often cast in general terms like 'improving patient care.' In her view, this is remarkable as the legitimacy of CES within healthcare institutions and society as a whole depends, among other things, on a clear formulation of its goals [16].

The international literature reveals a broad set of goals to be realized by CES. In different wordings scholars emphasize that CES should ultimately enhance the quality and goodness of care. For example, according to the ASBH-report [2, p. 8] the general goal of health care ethics consultation is to "improve the provision of health care and its outcome through the identification, analysis and resolution of ethical issues as they emerge in clinical cases in health care institutions." Aulisio et al. [3], for instance, indicate that ethics consultation aims to 'to help to identify and analyze the nature of the value conflict or uncertainty' (p. 61) and to 'forge consensus among involved parties.' More specifically authors stress that information exchange, communication, shared understanding and decision-making are goals of CES [14, 15]. Others emphasize the importance of moral reflection as a goal of CES $[1,13,18$, 19]. Reflection includes reflection on a case, reflection on what it means to be a good professional and reflection on institutional or organizational issues.

While ethicists specify goals of CES, it is largely unknown what kind of goals keypersons in healthcare institutions strive for when implementing CES. Some empirical studies cast light on the goals seen as important in practice. For instance, findings of a survey of Fox et al. [8] show that intervening to protect patients rights (94\%), resolving real or imagined conflicts $(77 \%)$, changing patient care to improve quality (75\%) and increasing patient/family satisfaction (68\%) were regarded as important goals of ethics consultation by US hospitals. Molewijk et al. [13] present the results of a study in which participants of moral case deliberation stated what they perceived as goals of moral deliberation. From a list of 15 different goals, the following goals were reported as most important: (1) to activate my job motivation, (2) to get knowledge of and insight in moral issues, (3) to pay attention to reasons and arguments and (4) to improve mutual understanding. Both these studies reveal important insights, but used a preordained questionnaire, which may have steered the answers.

The purpose of this article is to present results of a systematic, empirical research on what key-persons in Dutch healthcare institutions consider as goals of CES. We started with open ended questions for board members of all Dutch healthcare institutions as this allowed them present their goals. The presumption is that a better understanding of the goals of CES in practice may help ethicists to be more aware of the organizational context in which they operate, and provide a tool to foster a dialogue about the goals of CES among ethicists and practitioners in order to improve the implementation and evaluation of CES.

\section{Methods}

This investigation was part of a larger research project in which we used an integrated mixed methods design. Quantitative and qualitative methods were intentionally mingled during the research process for purposes of triangulation and 


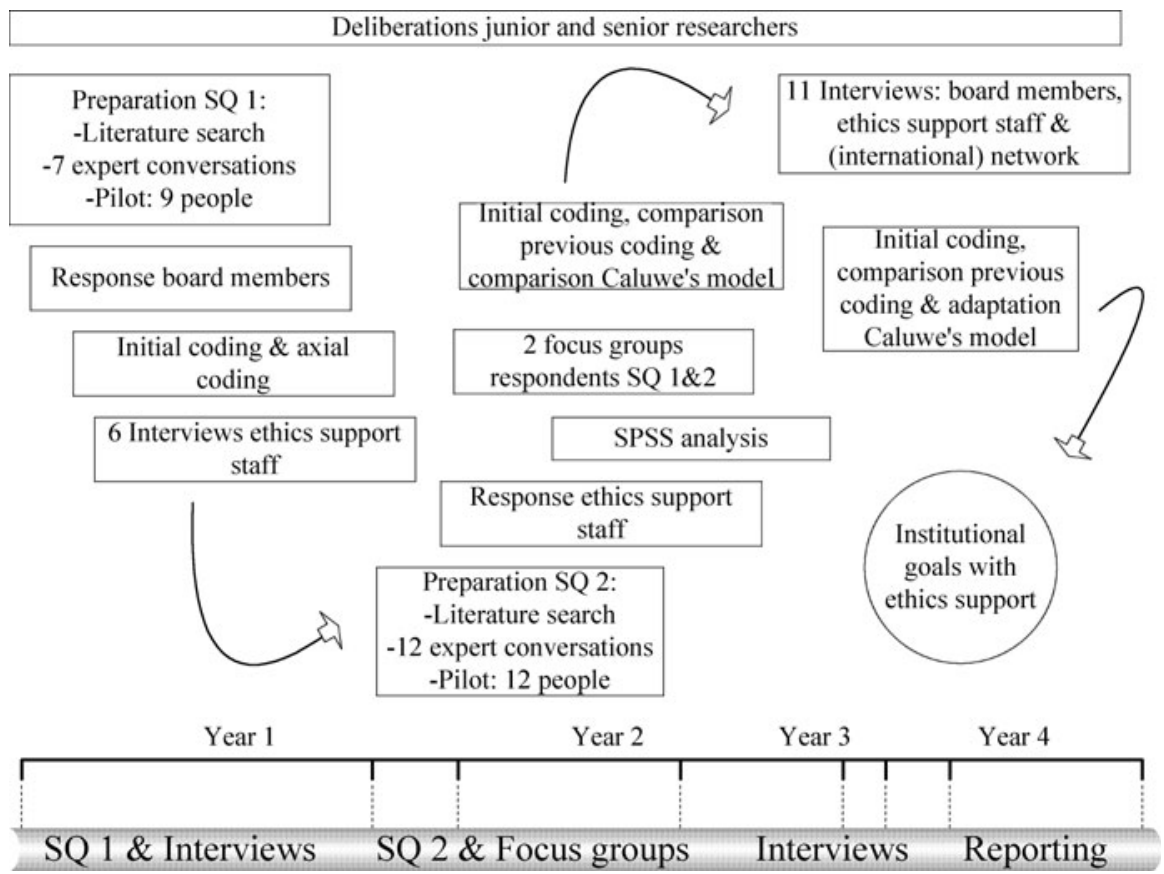

Fig. 1 Research flow: data collection and analysis

complementarity [12]. Research subjects were board members of all Dutch health care institutions, ethics support staff, indicated by the replying board member, and (inter) national advocates of CES. Ethics support staff refers to employees of a health care institution who organize and implement CES (such as chairs of ethical committees, or spiritual caregivers).

Figure 1 presents a detailed description of the research procedures followed.

Survey questionnaire 1 (SQ1) was directed at the board members of healthcare institutions. Its development has been described elsewhere in detail [7]. The main topics of the survey questionnaire were based on the short questionnaire of Slowther [17], pointing at needs and ways of CES. Among other things we added an openended question on the goals of CES. This (postal) questionnaire was sent to all intramural healthcare institutions $(\mathrm{N}=2137)$ registered with the Netherlands Ministry of Health, Welfare and Sports, including elderly care institutions, hospitals, mental healthcare and institutions for people with an intellectual disability. The response rate was $56 \%$. A possible reason for not answering SQ 1 is that the postal questionaire did not reach the right person as we did not have names of the board members. Furthermore, CES might not have the priority of board members. After the first analysis of questionnaire 1, we conducted 6 semi-structured interviews with ethics support staff members to further understand the initial analysis of questionnaire one including the first analysis of the goals and to prepare survey questionnaire 2. Respondents were selected by a national platform for CES. The interviews were recorded, analyzed, and returned for validation (member check). 
Survey Questionnaire 2 (SQ2) included questions that the board members were unable to answer in the pilot e.g. on content, participants, integration and evaluation of CES. The relationship between SQ1 and SQ2 was that they both investigated the need for, ways of and goals of CES in Dutch healthcare institutions. Different were the respondents (board members in SQ 1, ethics support staff in SQ 2), number of questions (more in SQ2) and the way of asking (open versus closed questions). For example, in SQ2, questions about the goals of CES were closed; the answering options were based on the first analysis of the responses to the open ended question about goals of CES in questionnaire 1. This (digital) questionnaire was sent to ethics support staff, indicated in the response to SQ1 $(\mathrm{N}=515)$. The response rate was $48 \%$ (247/515). A reason for not answering SQ 2 might be that the questionnaire was too long and respondents did not have enough time to complete it.

In addition two heterogeneous focus groups were organized. They were attended by a mix of board members/directors and ethics support staff. We constructed the focus groups with board members and ethics support staff because they are well informed and reflective. Further, our research also aimed to stimulate dialogue about CES with this target group on a national level as these respondents play an important role in facilitating and organizing CES. Participants received a mid-term report on the research prior to the meeting. The meeting itself was structured by the issues emerging from the survey questionnaires, and followed an agenda that left ample room for exploration and dialogue. Each two-hour session was moderated by the first and the last author. The conversations of the two focus groups were summarized and sent to the participants for validation (member check).

Aiming for corroboration and deeper understanding of the findings we conducted 11 additional semi-structured interviews in the third year of the overall research project. Themes again included goals of healthcare institutions concerning CES, needs for CES, and forms of CES. The interviews were recorded, analyzed, and returned for validation (member check).

\section{Analysis}

The data were first analyzed separately and then transformed for further analysis and comparison (crossover track analysis) [9]. The analysis of the qualitative data on goals of CES followed a thematic content analysis. There was an initial coding of the data in which we read each text part line by line and labelled it with codes. The codes of all data were then compared and clustered and renamed into new codes that covered all initial codes. In this phase we also made a detailed description of the initial codes. Then, we searched for relationships among the codes and visualised this in a first model (which finally led to Fig. 2). This model was discussed between the researchers in order to find the most appropriate codes/labels and clustering of the goals for CES. After incorporating their response these codes were used for the closed question about goals of CES in SQ2. The answers to the closed questions of SQ 2 were analyzed using SPSS 15.

The analysis was completed by the first author together with the research team. The combination of methods (triangulation procedure) added breadth to the study, 


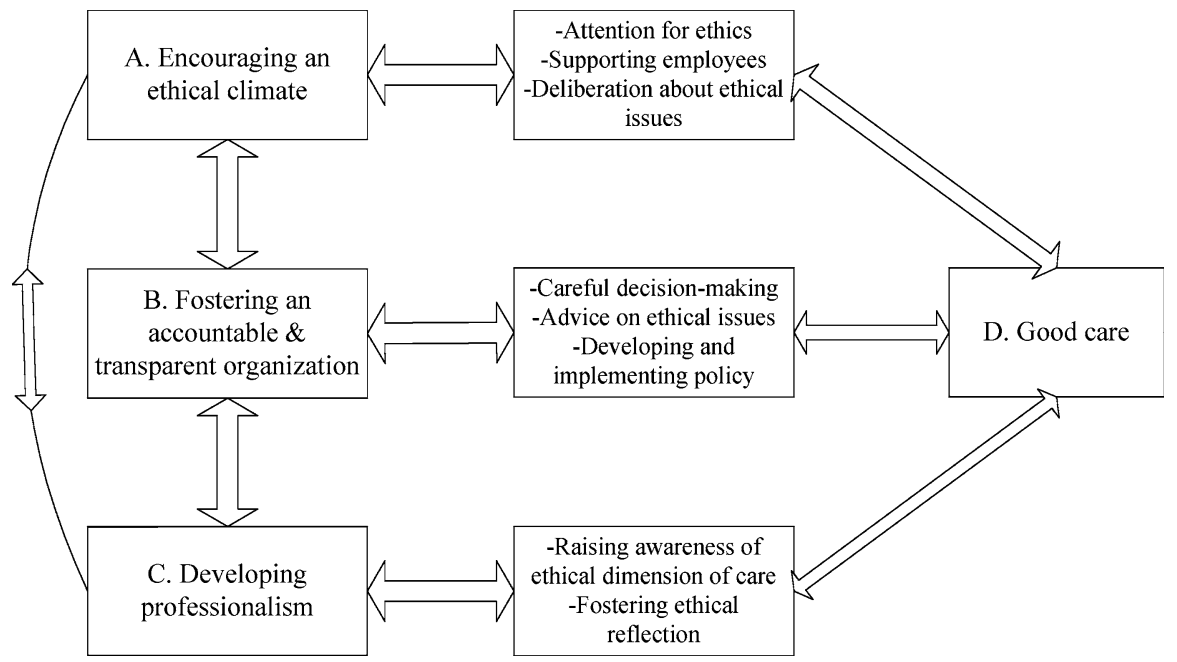

Fig. 2 Reported goals of health care institutions with Clinical Ethics Support

and the qualitative data provided explanations for the numeric data from the surveys.

\section{Results}

Respondents listed a very broad array of goals of CES in SQ1. In total we identified four main goals and eight sub-goals. Table 1 summarizes how ethics support staff (SQ2) valued different sub-goals of CES. ${ }^{1}$ The answering categories were based on the initial analysis of the responses to SQ1. Therefore they are not exactly the same as Fig. 2, which is the result of a more rigorous analysis. Respondents of SQ2 characterized the importance of each goal by indicating the goal as: '(absolutely) a goal' or '(absolutely) no goal.'

Table 1 shows that respondents of SQ 2 state that all sub-goals of CES in healthcare institutions are important. At least $72 \%$ the respondents indicate each sub-goal as (absolutely) a goal.

The goals that were most often mentioned as important were: attention for ethical issues (98\%), raising awareness of ethical aspects (97\%), fostering ethical reflection (95\%), improving quality of care (93\%) and supporting employees (92\%).

Respectively $17 \%$ and $26 \%$ of the ethics support staff indicated that 'to advice about ethical issues' and 'to make ethical policy' should (absolutely) not be a goal of CES.

The broad set of goals was clustered into a framework including four main goals of CES (see Fig. 2): (a) Encouraging an ethical climate (including), (b) Fostering an

\footnotetext{
1 There are no data available of how the board members valued the importance of the different goals since the categories were not identified at that time.
} 
Table 1 Reported sub-goals of ethics support staff with CES (SQ2, $\mathrm{n}=86$ )

\begin{tabular}{llll}
\hline Goals & $\begin{array}{l}\text { (Absolutely) } \\
\text { a goal (\%) }\end{array}$ & $\begin{array}{l}\text { (Absolutely) } \\
\text { no goal (\%) }\end{array}$ & $\begin{array}{l}\text { No } \\
\text { answer (\%) }\end{array}$ \\
\hline Attention for ethical issues & 98 & 2 & 0 \\
Raising awareness of ethical aspects & 97 & 3 & 0 \\
Fostering ethical reflection & 95 & 5 & 0 \\
Supporting employees & 92 & 6 & 2 \\
Systematic deliberation on ethical issues & 84 & 14 & 2 \\
To promote decisions with an ethical & 81 & 14 & 5 \\
$\quad$ dimension & & 17 & 5 \\
To advice about ethical issues & 78 & 26 & 2 \\
To make ethical policy & 72 & & \\
\hline
\end{tabular}

accountable and transparent organization, (c) Developing professionalism and (d) Good care.

Encouraging an Ethical Climate

Key-persons in healthcare institutions see CES as a vehicle to encourage an ethical climate within the organization. This entails creating an environment in which reflection on ethical issues is stimulated. This implies that ethics is a part of the organizational culture, as an everyday issue. CES facilitates an ethical climate by: (1) attention for ethics; (2) supporting employees and (3) deliberation about ethical issues.

\section{Attention for Ethics}

Encouraging an ethical climate includes attention for ethics. Attention for ethics was considered as a very important goal of CES among a large part of the respondent (98\%). Healthcare institutions see CES as a means to pay attention to ethical issues continuously. "Make [employees] enthusiastic about ethical dilemmas."(Respondent SQ1) and "Disseminate ethics broadly." (Respondent SQ1).

\section{Supporting Employees}

Employees have to deal with moral questions daily, and healthcare institutions see CES as a means to cope with these questions by providing specific methods and knowledge. Supporting employees is considered a highly important goal of CES among the respondents (92\%). It is acknowledged that especially employees on the work-floor are often in a difficult position and need support to be able to handle moral dilemmas. The perceived surplus value of CES varies from stimulating an enhanced understanding of cases in daily practice to improving the satisfaction and comfort of employees: 
A feeling of safety and the satisfaction of employees, both physicians and nurses. For me that's a very important outcome (Interviewee, Director knowledge centre, mental health care)

\section{Deliberation About Ethical Issues}

Health care institutions see CES as a means to facilitate deliberation about ethical issues in daily practice. Respondents indicate that CES should initiate or facilitate (systematic) conversation about ethical issues in the organization; to create an "Opportunity to talk about choices and dilemmas in our work." (Respondent SQ1) This implies that there is an open dialogue between all stakeholders, characterized by equality and asking questions, in order to understand the perspectives of the other. Through deliberation, ethical issues become a part of the organizational culture: "To promote a climate in our residence in which it is possible to talk about ethical issues." (Respondent, SQ1) Addressing ethical issues should be an everyday activity: "It [moral deliberation] is just a tool for conversation" (Moral deliberation conversation leader, mental health care). Deliberation is regarded as a means to make the organization work on a dialogical basis.

Influencing processes and relationships so people are able to talk with the client or family, but also to have dialogues with each other (network organization elderly care institutions)

Fostering an Accountable and Transparent Organization

Healthcare institutions use CES to foster an accountable and transparent organization. This means that CES is seen as a vehicle to express and (re)shape their accountability. CES can help health care institutions to be an accountable organization by developing and implementing an institutional policy (including core values of the organization). Respondents mention the following sub-goals: (1) Careful decision-making; (2) Advice about ethical issues and (3) Developing policy.

\section{Careful Decision-making}

Respondents indicate that within an accountable organization stakeholders are able to make careful and justified decisions concerning ethical issues. CES is considered as a vehicle to realize the goal of careful decision-making. For example:

We need to take responsibility for the choices we make. Choices in treatment techniques or diagnostic activities. Or: what kind of patients do we particularly want to treat here? These kinds of choices should be based on argumentation. In my view an ethical discussion about this, is an important contribution to these arguments (interviewee, chair of the board, hospital).

This implies that CES facilitates stakeholders to motivate and take responsibility for their actions, and to be accountable for actions. CES stimulates practitioners to 
develop and express their professional accountability regarding their decisions, actions and view points.

\section{Advice About Ethical Issues}

This means that an expert gives advice (asked and unasked) on ethical issues which arise in daily practice. Advice can be either general, or focus on concrete cases. The quantitative data show that advice is not univocally considered as an important goal of CES. The qualitative data explain that advice has benefits in terms of leading to a shared perspective and/or solution of a day-to-day situation:

Advice can facilitate collaborative suggestions/bring a fast solution. For example a woman with dementia who urinates in her bed. Together you can come to a certain frame, you can look at the situation from different perspectives (participant FG).

However, respondents indicate that a focus on advice might make CES a matter of experts, having a high threshold:

It will be a product of a small group, with too much focus on principles. It could be a base for reflection and learning but should stimulate professionals to think themselves' (Participant FG)

\section{Developing and Implementing Policy (On Ethical Issues)}

Respondents indicate that CES can facilitate policymaking within healthcare institutions as it invites stakeholders to express what they see as important (core) values for the organization. Respondents see CES as a way to formulate a mission or vision: 'To develop guidelines and vision' (Respondent SQ1). More specifically, CES can help healthcare institutions to develop policy on how to deal with ethical issues within their organization: "We only have one main goal and that's the agenda setting of ethics on all units, all levels and within all layers of the organization." (Interviewee, Medical ethicist hospital). CES can also help implementing policy throughout the organization. This implies that the vision on quality is diffused within all layers of the organization. Respondents indicate that CES connects rules to practice and helps to better apply existing guidelines. For example:

As organization we think it is important to implement the principle of recovery. It is a challenge because: 'we, with a small group, might think it is important. But there are about 500-600 people working here for this target group and they all have a practice.' In order to implement this, we should have dialogue. And than moral deliberation is a method (Director knowledge centre, mental health care).

Some respondents indicate that ethical justification is a goal of CES. "Being univocal to the external world." (Respondent SQ1) and "Transparency of ethical issues" (Respondent SQ1). 
Respondents see policymaking not as one of the more important goals of CES. They seem to be a bit sceptical about the instrumental value of CES as a vehicle to realize organizational goals as this might decrease the free space for reflection and dialogue. Some respondents consider CES as an enclave within the organization that becomes more and more driven by economic values and business concepts. If CES is tied up with policy goals and strategic action, they fear that it will lose its free conversation space and communicative action.

A cultural change is needed to achieve the goal of deliberation about ethical issues. It should be supported by the whole healthcare institution and the culture should not be too business-like (Participant FG).

\section{Developing Professionalism}

A third main goal of health care institutions with CES is developing professionalism. This is an educational goal. It entails that practitioners are able to recognize ethical questions and are able to deal with them. Sub goals of developing professionalism are: (1) Raising awareness of the ethical dimensions of care and (2) Fostering ethical reflection.

\section{Raising Awareness of the Ethical Dimensions of Care}

Respondents assume CES will raise the awareness of the ethical dimensions of care among employees. Awareness of ethical issues gained high priority (97\%). Respondents assumed that through CES practitioners will increasingly become sensitive for and able to recognize ethical aspects in daily health care practice: "To improve the capacity of employees to recognize ethical aspects of care." (Respondent SQ1). Another interviewee explains what awareness means in elderly care:

Most important is the awareness that you (as caregiver) make ethical choices everyday. For example: will you tell a daughter of a mother with dementia that she has a relationship with another patient? (Head of ethics committee, nursing home)

Raising awareness is considered as an extremely important goal of CES. This is related to the concern that employees not always recognize their responsibilities concerning the ethical dimensions of their work:

Each healthcare professional should think about the good and bad of actions. I am worried about ethical reflection of professionals and have the idea they execute what is already decided and documented by others and that the appeal on their own responsibility is decreasing. Professionals have the idea they cannot influence (organizational) choices anymore. Care is, however, about unique people and when there is no appeal on the individual assessment capacity of professionals anymore, than this damages the profession (Director of Christian association for health care authorities). 


\section{Fostering Ethical Reflection}

Developing professionalism also means stimulating and fostering reflection. This is considered as an extremely important goal of CES (95\%). This implies that practitioners develop attitudes and skills which make them able to (systematically) reflect upon moral situations and moral questions. Reflection results in looking differently at a situation, for example by asking oneself: 'Am I patient enough?' Ideally, CES should stimulate reflection in daily practice:

That you, as a matter of fact, develop a routine to think in a concrete situation, but also afterwards: 'what we did, was it good or should we have acted differently? (interviewee, chair of the board, hospital).

One interviewee has an advanced idea of three levels of reflection and states that CES facilitates reflection on a deeper level, namely the second and third level.

There are different levels of reflection. First, very basic, e.g.: 'My goal is to do all 17 beds before the end of the morning.' At the end I assess: 'did I make it?' No, again there was a lack of time at the end. Why is that? I can reflect on that critically. A second level of reflection asks: 'is it desirable that I want to reach this goal in this way? Shouldn't we organize work another way?' A third, deeper form of reflection is: what does this say about the care we want to offer? The way we deal with it. These levels are also denominated as: primary, secondary and tertiary reflection or single loop, double loop and triple loop learning (Director of Christian association for health care authorities)

Reflection is considered as an important goal. This is related to the lack of reflection that is signalled in the current healthcare context:

Employees work in a very hectic environment, that's unbelievable. Often they don't have time to reflect on situations. When we are called in, then we do reflect, it is a moment of reflection, consideration. Like: 'we don't do it that bad' or 'it could be different' or 'it's not strange that I think that' or 'she also has it.' Yes, that's really a surplus value (interviewee, member of ethics committee, nursing home).

\section{Good Care}

Good care is the fourth main goal healthcare institutions have with CES. They want to deliver care in concordance with the needs of patients, given available means. Participants explain that good care does not have to be based on principles. The determination of good care depends on contextual factors, and has a different meaning for the stakeholders who define it. Divergent views on quality of care are not regarded as a problem. Rather, communication is required to come to a joint perspective: CES is a means to facilitate that kind of communication.

There is no definition of what good care is. Questions and answers should converge and a common view should develop in interaction (Participant FG). 
Some stress that CES should and will add to the quality of the relationship between caregiver and care-receiver. These respondents refer to the continuous, contextual and situational process which is characteristic for questions about what is good.

The more fundamental, intense and long term care is, the more you desire quality of care in that relationship. Consequently, more is required from a professional: to asses the situation time and time again and ask oneself the question: what's, for this human being, in this situation, today good care? (Director of Christian association for health care authorities)

It is expected that CES can help practitioners to develop ethical competences like a good attitude to patients:

That's also ethics: 'I just want to do my job.' But what's that 'just?' Do you come and do your business and than exactly at five a clock you close and put the answering machine on? Or do you work with inspiration? And do you engage with the patient: 'no matter what happens, this patient shouldn't be waiting longer than necessary.' I am kind and work 5 min longer. It is about that kind of ethics. Why is it so important to put ethics on all these agendas? because we expect that when you address ethical competences and train people in these, attitudes and treatment towards clients will be changed (Medical ethicist, hospital)

Good care and (improving) quality of care are used as synonyms by respondents. Improving quality of care for example means that the care actions (process), the organization and the care delivered (output) are qualitative high. Some stress that CES should be a part of internal quality processes:

We trained 25 people to develop ethical qualities within the organisation. They facilitate the dissemination of this knowledge and try to set up dialogues about ethical dimension of care. This is a part of internal quality improvement. (Director knowledge centre mental health care institution)

Figure 2 also shows the interrelation between the goals of CES in health care institutions. It indicates that health care institutions see 'good care' as the ultimate goal of CES. Basically, participants of our study indicate that all (main- and sub) goals with CES contribute to the overall goal: 'good care.'

\section{Discussion}

Our findings illustrate that respondents see good care as the overall goal of CES (see Fig. 2) and this is confirmed by previous literature. Authors use different words, for example: improving patient care [16], to improve the quality of care [1], delivering good care [19] and facilitating good life [4, 5]. Some authors argue that good care or good life should be the overall goal of care and see CES as a concrete way to realize this overall goal of care [5]. Others say good care is a goal much too vague and in need of operationalization [16]. This raises the question to what extent they see good care as a distinguishing parameter of CES. 
For managerial reasons it might indeed be helpful to operationalize the general goal of good care into smart targets. Such targets focus attention and enable managers to evaluate the effectiveness of CES. Yet, such concretizations should fit with the goals of employees, and general goals should not be neglected as they form motivators for CES in practice. Our findings indicate that participants in practice stress the link between the higher and ultimate goal of good care, and other goals and sub-goals which are considered as intermediate goals to realize the higher goal of good care. Thus, for example, supporting employees may add to their professionalism, and this may lead to better care. Likewise, for instance, deliberation may improve the quality of care. Such common sense relations are intuitive and hypothetic, but still extremely important as motivators for CES. One might say that good care is the driving motivator behind CES in practice, and we argue that this ultimate goal - the answer to the question why we are doing all this-is extremely important to encourage people to participate into the whole project of CES. People need a higher ideal which resonates with their own and organization goals to be able to join into the idea of CES.

Our findings indicate that in healthcare practice attention for ethical issues, awareness of ethical issues, fostering ethical reflection and supporting employees are considered important sub-goals of CES. These sub-goals are part of the main goals encouraging an ethical climate and developing professionalism. An ethical climate has been defined as 'the organizational practices and conditions that promote discussion and resolution of decisions with ethical content' [10]. It has been demonstrated that supporting employees helps to create a better ethical climate as it lowers moral distress [10]. Previous literature also confirms the importance of developing professionalism by CES. For example, the sub-goal reflection is described by Bolmsjo et al. [4], Svantesson et al. [18], Abma et al. [1] and Vanlaere et al. [19]. Our findings suggest that particularly these goals (ethical climate, reflection) might play an important role in the practical use, implementation and evaluation of CES. We assume, however, that the importance endowed to these (sub-)goals is at least in part influenced by the context of our study. In The Netherlands, where this study was completed, reflection and dialogue are considered highly important. This might be an aspect of the organizational culture of healthcare institutions, and our societal culture more in general, which can be characterized as being egalitarian [11]. Our egalitarian culture fits with the value endowed to democratic dialogue and deliberation, and the preference for moral case deliberation as the preferred form of CES. Our culture also explains why the goal of giving advice was not met with enthusiasm. Advice implies more hierarchy, and acceptance of expert knowledge. This implies that our findings cannot just be generalized to other contexts with a less egalitarian culture.

The model with four clusters of goals of CES indicates that in order to realize the higher goal of good care, healthcare institutions have to work on different domains ranging from developing an ethical climate and stimulating professionalism to fostering a responsible organization. We would argue that in order to make the implementation of CES feasible it is wise to choose one of the clusters of goals to start with. Ideally the choice for such a cluster of goals is the product of discussions between members of ethics staff, managers and practitioners within the 
organization, as this will foster the commitment for CES. The goal of CES should also fit with the culture of the organization. This implies that ethicists should beware of the organizational context they work in, and not just imply or impose their goal on the organization. Caluwé and Vermaak [6] developed a typology of organizations which might be helpful to match goals of CES with the organization context. For example, blue print organizations are characterized by (rational) planning and control. Such types of organization might want to choose to work on the goal of being an accountable and transparent organization. Green print organizations, on the other hand, value learning. Green refers to growth (as in nature) and 'green light,' as the objective is to get peoples' ideas to work. Green organization cultures might prefer to work on professionalism as a goal of CES. White print thinking assumes everything is changing autonomously. White reflects all colours and it denotes openness as it allows room for self-organization and evolution. The goal of fostering an ethical climate fits well with white print organization cultures. When working in a white print organization, advocates of CES do not necessarily have to define the goals of CES beforehand because the characteristic of this kind of organization is that it is not possible to plan everything beforehand.

A limitation of this study is that we could not include the views of caregivers and care receivers on the goals of CES in healthcare institutions. However, our respondents-board members and ethics support staff-play an important role in the practical use, implementation and evaluation of CES. Furthermore, there might be a positive bias in the sense that people who participated in our study might be more interested in ethics then non-responders who could have other or no goals with CES.

\section{Conclusion}

This article illustrated which goals key-persons in Dutch healthcare institutions attach to CES. We recommend that advocates of CES take into account the perceptions in healthcare organisations and distinguish main- and sub-goals, in order to promote the practical use, implementation and evaluation of CES in health care institutions. Our model can be used as a tool to foster dialogues about goals between various participants in healthcare institutions. The ultimate goal of good care should be valued as the driving motivator for CES in practice. The intermediate goals of CES (ethical climate, accountable organization, professionalism) should be connected to the characteristics of the organization culture as a whole. Taking into account the fit between the underlying values of CES and organizational culture will foster the acceptance of CES within organizations and add to the effectiveness of CES. This should not be organized by ethics support staff individually; the choice for intermediate goals fitted to the organization is ideally the outcome of a dialogue between the top of the healthcare institutions, advocates of CES and practitioners, as this fosters commitment for CES. We recommend further empirical research into the goals of CES. Especially, the goals of CES which are relevant for caregivers and care receivers, should be subject of deeper investigation. Finally, we recommend further research into the extent in which the goals for CES are congruent with the 
organizational culture and to what extent this congruency influences the success of CES. Although goals of CES tend to be broad and general, they nevertheless have a strong motivating power, and awareness of a variety of goals related to the cultural context can help integrating CES within healthcare institutions.

Acknowledgments We would like to thank all participants of the research project for their openness and effort. A special thank for the Department of Ethics at the Dutch Ministry of Health Welfare and Sports who sponsored the research.

Open Access This article is distributed under the terms of the Creative Commons Attribution Noncommercial License which permits any noncommercial use, distribution, and reproduction in any medium, provided the original author(s) and source are credited.

\section{References}

1. Abma, T. A., Molewijk, B., \& Widdershoven, G. A. M. (2009). Good care in ongoing dialogue. Improving the quality of care through moral deliberation and responsive evaluation. Health Care Analysis, 17, 217-235.

2. ASBH Task Force on Standards for Bioethics Consultation. (1998). Core competencies for health ethics consultation. The Report of the American Society for Bioethics and Humanities. ASBH.

3. Aulisio, M. P., Arnold, R. M., \& Youngner, S. J. (2000). Health care ethics consultation: nature, goals, and competencies. Annals of International Medicine, 133, 59-69.

4. Bolmsjö, I. Å., Sandman, L., \& Andersson, E. (2006). Everyday ethics in the care of elderly people. Nursing Ethics, 13(3), 249-263.

5. Bolmsjö, I. A., Edberg, A. K., \& Sandman, L. (2006). Everyday ethical problems in dementia care: a teleological model. Nursing Ethics, 13(4), 340-359.

6. Caluwé, L., \& de Vermaak, H. (2003). Learning to change. A guide for organization change managers. Thousand Oaks: Sage Publications.

7. Dauwerse, L., Abma, T., Molewijk, B., Widdershoven, G. (2011). Need for ethics support in healthcare institutions: Views of Dutch board members and ethics support staff. Journal of Medical Ethics. doi:10.1136/jme.2010.040626.

8. Fox, E., Myers, S., \& Pearlman, R. (2007). Ethics consultation in United States hospitals: A national survey. The American Journal of Bioethics, 7(2), 13-25.

9. Greene, J. C. (2007). Mixed methods in social inquiry. San Francisco: Wiley aand Sons.

10. Hamric, A. B., \& Blackhall, L. J. (2007). Nurse-physician perspectives on the care of dying patients in intensive care units: Collaboration, moral distress, and ethical climate. Critical Care Medicine, 35(2), 422-429.

11. Hofstede, G. (2001). Cultures consequences comparing values, behaviors, institutions and organizations across nations. Thousand Oaks: Sage publications.

12. Mertens, D. M. (2010). Research and evaluation in education and psychology. Integrating diversity with quantitative, qualitative and mixed methods. Washington DC: Sage publications.

13. Molewijk, B., Verkerk, M., Milius, H., \& Widderhoven, G. (2008). Implementing moral case deliberation in a psychiatric hospital: Process and outcome. Medicine, Health Care and Philosophy, $11,43-56$.

14. Racine, E., \& Hayes, K. (2005). The need for a clinical ethics service and its goals in a community healthcare service centre: A survey. Journal of Medical Ethics, 32, 564-566.

15. Reiter-Theil, S. (2001). The Freiburg approach to ethics consultation: Process, outcome and competences. Journal of Medical Ethics, 27, 21-23.

16. Slowther, A. (2007). Ethics consultation and ethics committees. Ch 72 in: Principles of health care ethics (pp. 527-532). England: John Wiley and Sons.

17. Slowther, A., Bunch, C., Woolnough, B., \& Hope, T. (2001). Clinical ethics support services in the UK: An investigation of the current provision of ethics support to health professionals in the UK. Journal of Medical Ethics, 27, 2-8. 
18. Svantesson, M., Löfmark, R., Thorsén, H., Kallenberg, K., \& Ahlström, G. (2008). Learning a way through ethical problems: Swedish nurses' and doctors' experiences from one model of ethics rounds. Journal of Medical Ethics, 34, 399-406.

19. Vanlaere, L., Coucke, T., \& Gastmans, G. (2010). Experiential learning of empathy in a care-ethics lab. Nursing Ethics, 17(3), 325-336. 